\title{
Correction to: Social exclusion of older persons: a scoping review and conceptual framework
}

\author{
Kieran Walsh $^{1} \cdot$ Thomas Scharf $^{2} \cdot$ Norah Keating $^{3,4}$
}

Published online: 26 July 2018

(c) Springer Nature B.V. 2018

\section{Correction to: Eur J Ageing (2017) 14:81-98 https://doi.org/10.1007/s10433-016-0398-8}

The article Social exclusion of older persons: a scoping review and conceptual framework, written by Kieran Walsh, Thomas Scharf, Norah Keating, was originally published electronically on the publisher's internet portal (currently SpringerLink) on 11 October 2016 without open access.

With the author(s)' decision to opt for Open Choice the copyright of the article changed on June 2018 to
(C) The Author(s) 2018 and the article is forthwith distributed under the terms of the Creative Commons Attribution 4.0 International License (http://creativecommons.org/licenses/ by/4.0/), which permits use, duplication, adaptation, distribution and reproduction in any medium or format, as long as you give appropriate credit to the original author(s) and the source, provide a link to the Creative Commons license and indicate if changes were made.

The original article can be found online at https://doi.org/10.1007/ s10433-016-0398-8.

Kieran Walsh

kieran.walsh@nuigalway.ie

1 Irish Centre for Social Gerontology, National University of Ireland Galway, Galway, Ireland

2 Institute of Health \& Society, and Newcastle University Institute for Ageing, Newcastle University, Newcastle, UK

3 Centre for Innovative Ageing, Swansea University, Swansea, UK

4 Africa Unit for Transdisciplinary Health Research (AUTHeR), North-West University, Potchefstroom, South Africa 